\title{
Desartrodesis de tobillo, experiencia en México. Reporte de dos casos
}

\section{Ankle arthrodesis takedown, Mexico's experience. Two cases report}

\author{
Luis A Gómez Carlín, ${ }^{*}$ Luis Felipe Hermida Galindo, ${ }^{\ddagger}$ Santiago Guerrero§
}

Citar como: Gómez CLA, Hermida GLF, Guerrero S. Desartrodesis de tobillo, experiencia en México. Reporte de dos casos. An Med (Mex). 2021; 66 (1): 48-53. https://dx.doi.org/10.35366/99489

\section{RESUMEN}

Se presentan dos casos clínicos de artrosis de tobillo que fueron tratados con artrodesis tibioastragalina. En el primero la artrodesis fue exitosa; el paciente se presentó con una inestabilidad subastragalina que provoca los síntomas al paciente; mientras que en el segundo, se trató de una pseudoartrosis de tobillo que se manifestaba con dolor medial de tobillo, lo que limitaba la función ambulatoria del paciente. En ambos casos se realizó la conversión a una prótesis total de tobillo con buenos resultados. La conversión de una artrodesis de tobillo hacia una artroplastia total del mismo es un concepto relativamente nuevo que ofrece la alternativa de sumar movilidad en el tobillo y, sobre todo, disminuir las cargas de las articulaciones vecinas que evitarán una artrosis secundaria. Aunque es baja la incidencia de este procedimiento por cada cirujano, se está formando una buena cantidad de experiencia a través de la complicación de casos en grupos organizados para evidenciar su eficacia en casos determinados. Dentro de los más comunes están la conversión por una artrodesis fallida, ya sea por pseudoartrosis o mala consolidación y por trastornos de la subastragalina posterior a una artrodesis de tobillo.

Palabras clave: Conversión de artrodesis de tobillo, artrodesis de tobillo, artroplastia total de tobillo.

\section{ABSTRACT}

Two cases of ankle osteoarthritis that were treated with tibiotalar arthrodesis are presented. In the first case the fusion was successful but a gross sub-talar instability developed that gave the patient symptoms and in the second patient, a non-union occurred and progressive medial pain was the patient chief complaint. In both cases, a total ankle arthroplasty was made with a very good outcome. Ankle arthrodesis conversion to a total ankle arthroplasty is new concept that offers not only a regain in ankle range of motion but also the prevention of early secondary arthrosis of the neighbor joints by unloading them, specially the sub-talar joint. Although this is not frequent procedure for each surgeon, cases are being compilated by groups in order to have a solid evidence of its efficacy in cases like an ankle non-union/ mal-union, sub-talar disease posterior an ankle fusion.

Keywords: Ankle arthrodesis conversion, ankle arthrodesis, total ankle replacement.
* Ortopedia y Traumatología de Pie y Tobillo. Jefe de la Clínica de Pie y Tobillo Medyarthros. Presidente de la Sociedad Mexicana de Cirugía de Pie y Tobillo A.C, Guadalajara, Jal., México. Profesor Titular del Curso de Alta Especialidad en Cirugía de Pie y Tobillo por la Universidad de Guadalajara.

‡ Ortopedia y Traumatología de Pie y Tobillo. Ex Presidente de la Sociedad Mexicana de Cirugía de Pie y Tobillo A.C. Staff Centro Médico ABC, CDMX, México.

$\S$ Instructor de Pie y Tobillo Hospital Universitario de San José, Práctica Privada en Santa Ana Medical Center, Bogotá Colombia.

\section{Recibido: 10/01/2021.}

Aceptado: 20/03/2021.

Correspondencia: Dr. Luis F Hermida Galindo Av. Carlos Fernández Graef Núm. 154, consultorio 406, Santa Fe, Contadero, 05330 Cuajimalpa de Morelos, CDMX, México. Tel. 55 1664-7149

E-mail: drlfhermida@ hotmail.com 


\section{INTRODUCCIÓN}

Aunque el tobillo es la articulación mayor con menos incidencia de artrosis, ${ }^{1}$ llega a afectar a $1 \%$ de la población mundial. ${ }^{2}$ A diferencia de la osteoartrosis (OA) de la cadera y la rodilla, el tobillo rara vez tiene una causa primaria, siendo la OA postraumática la más frecuente, con reportes de 78 a $85 \%$ del total de los casos. ${ }^{3}$ Dentro de las causas postraumáticas, los principales factores de riesgo son las fracturas con mecanismo rotacional, seguidas de las lesiones ligamentarias (entorsis repetitivas). ${ }^{4,5} \mathrm{~A}$ su vez, las lesiones por entorsis repetitivas (por ejemplo, las producidas en el fútbol soccer) se relacionan a OA con varo de retropié. ${ }^{6}$ Ambos factores favorecen la incidencia de inestabilidad sub-talar. ${ }^{7,8}$ Por otra parte, es importante mencionar que la mayoría de los pacientes con OA postraumática son jóvenes. ${ }^{2}$

La articulación sub-talar, junto con el tobillo, forman la unidad funcional del retropié y por su compleja anatomía desempeña un papel muy importante en la regulación biomecánica del pie. ${ }^{7,8}$ Ambas articulaciones pueden presentar OA, la cual es una enfermedad crónica incapacitante, que se ha convertido en un importante problema de salud global. ${ }^{2}$ Incluso, algunos autores indican que en escalas de función física autorreportadas por los pacientes, la OA de tobillo se puede comparar con otras patologías como enfermedad terminal renal, insuficiencia cardiaca congestiva, dolor cervical o radiculopatías. ${ }^{9}$
Hasta hace poco, la artrodesis fue considerada el tratamiento estándar de oro; ${ }^{2}$ sin embargo, en la actualidad, la artroplastia total de tobillo ha demostrado mejores resultados postquirúrgicos representados en la función y en la calidad de vida del paciente. ${ }^{10-12}$

Aquellos sometidos a artrodesis de tobillo refieren satisfacción con el tratamiento, a pesar de las limitaciones funcionales resultantes. ${ }^{1,11,13}$ No obstante, el riesgo de presentar OA en articulaciones adyacentes es de hasta $90 \%$ en el primer año, ${ }^{1}$ a causa de un incremento en la movilidad de la articulación subastragalina en su faceta posterior de aproximadamente $11 \%$, por tanto, la OA subastragalina es una de las complicaciones más reportadas. ${ }^{11,13,14}$ Hasta el momento, no se ha establecido si esta OA temprana es secuela de lesiones ligamentarias talo-calcáneas, del ligamento fíbulo-calcáneo previas o son resultado del incremento en la rotación sub-talar posterior a la artrodesis del tobillo.

Existe una amplia gama de tratamientos quirúrgicos para corregir las complicaciones resultantes de una artrodesis de tibio-talar, dentro de las que se incluye la conversión de artrodesis a artroplastia total de tobillo. ${ }^{11,13,14}$ Por lo general, dicho procedimiento se acompaña de otros gestos quirúrgicos como osteotomías complementarias, artrodesis de articulaciones adyacentes, estabilización ligamentaria o liberación de tejidos blandos, entre otros.

Las indicaciones para convertir una artrodesis de tobillo a una artroplastia total son: una pseudoartrosis o mala consolidación de tobillo dolorosa, artrodesis

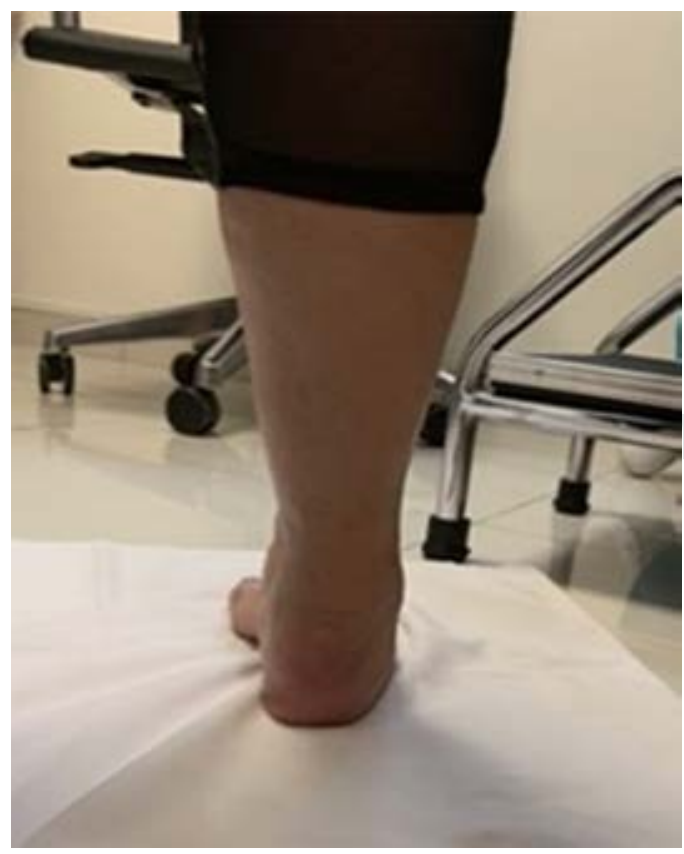

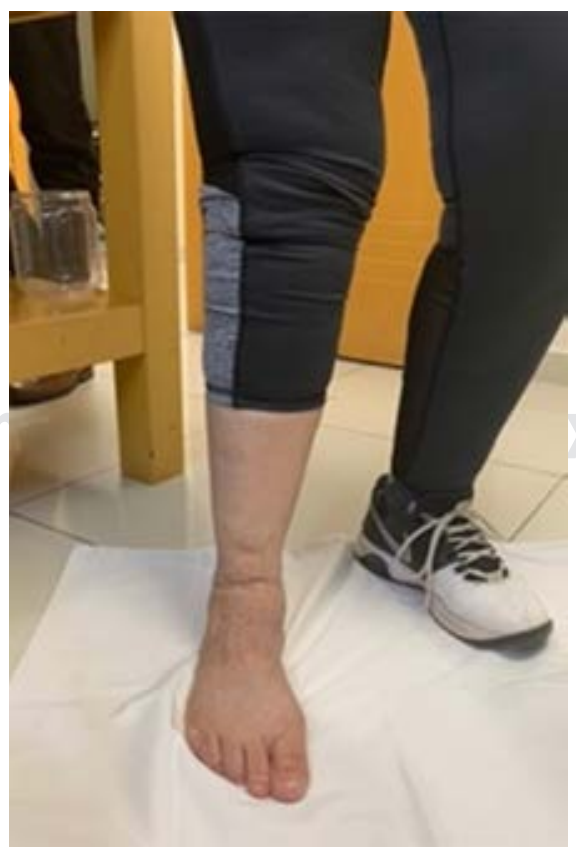

Figura 1:

Imágenes clínicas preoperatorias con varo, aducto y equino. 
Figura 2:

Radiografía anteroposterior y lateral del tobillo que demuestra la fusión y franca inestabilidad subastragalina.
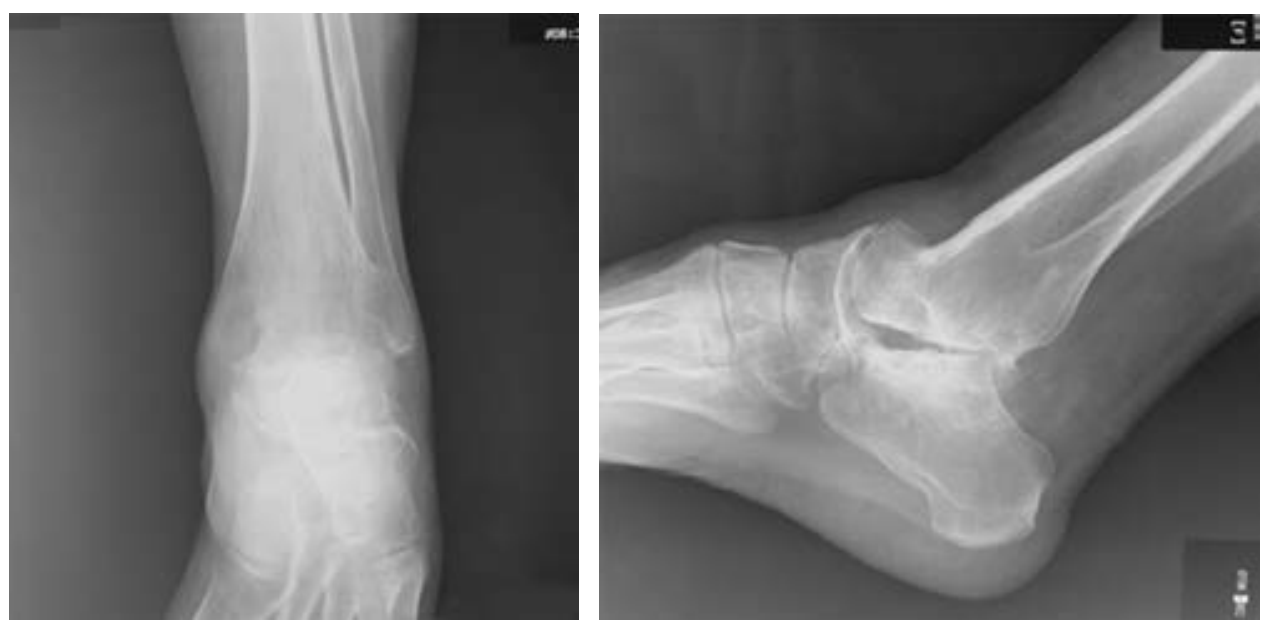

tibio-talar con artrosis dolorosa en la subastragalina y/o en la talo-navicular y una pseudoartrosis subastragalina en una artrodesis tibio-astrágalo-calcánea. ${ }^{4}$

\section{PRESENTACIÓN DEL CASO 1}

Paciente del sexo femenino de 56 años de edad, con antecedente de artrodesis de tobillo de 22 años de evolución. Presentó dolor, dificultad para la marcha, deformidad en equino del pie no reductible pasivamente, varo del retropié y recurvatum compensatorio de la rodilla (Figura 1).

El examen radiográfico mostró una consolidación completa de la artrodesis del tobillo y en neutro en los planos sagital y coronal, así como osteofito dorsal de la articulación talo-navicular y apertura anormal de la articulación sub-talar en la proyección sagital. En la vista anteroposterior se evidenció aducto del antepié a expensas de la articulación talo-navicular y cambios artrósicos incipientes (Figura 2).

Con estos hallazgos clínico-radiográficos se decidió realizar la conversión de la artrodesis tibio-talar a un reemplazo total de tobillo.

\section{Procedimiento quirúrgico}

Bajo anestesia regional, en decúbito dorsal e isquemia neumática, se practicó abordaje dorsal al tobillo, se incidió retináculo extensor con medialización del tendón tibial anterior y lateralización del extensor largo de los dedos junto con el paquete neurovascular del tibial anterior; se identificó el extremo distal de la tibia y los maléolos; se extendió distalmente el abordaje, identificando el cuello del astrágalo y la articulación talo-navicular; con visión fluoroscópica, se identificó la altura de lo que correspondería a la articulación talo-navicular y a las goteras medial y lateral del tobillo. A continuación, se realizó el procedimiento de acuerdo a la técnica quirúrgica habitual. La posición adecuada del implante protésico de prueba se verificó con fluoroscopia; posteriormente, se colocaron los implantes definitivos. Para la estabilización de la articulación sub-talar se utilizó un sistema de fijación flexible con botones siguiendo la dirección del ligamento cervical (Figura 3).

\section{PRESENTACIÓN DEL CASO 2}

Paciente masculino de 61 años de edad con dos cirugías previas en el tobillo derecho. La primera fue una artroscopia de tobillo para limpieza articular con microfracturas por una lesión osteocondral medial y un año después una artrodesis tibio-astragalina con dos tornillos. Un año posterior a la artrodesis, y pese a que su evolución había sido satisfactoria en cuanto al dolor los primeros 12 meses, el paciente inicia con dolor medial progresivo con caminatas de larga distancia. Clínicamente no había arco de movilidad del tobillo y dolor moderado a severo a la palpación de la superficie medial a nivel del receso. Se le propone desmantelar la artrodesis y colocar una prótesis total de tobillo derecho.

Radiográficamente se observa consolidación parcial de la artrodesis de tobillo con una adecuada alineación sin datos de aflojamiento de los tornillos (Figura 4).

\section{Procedimiento quirúrgico}

Bajo sedación y bloqueo poplíteo por ultrasonido con torniquete en muslo, se realiza abordaje ante- 
rior de tobillo de $8 \mathrm{~cm}$ entre el tendón tibial anterior y el extensor largo del hallux. Se hace el retiro de dos tornillos canulados de $6.5 \mathrm{~mm}$ y se observa que en el receso medial no había consolidación, sino una franca pseudoartrosis, mientras que en la zona anterior sí había consolidación. Se preparó la articulación con cinceles tratando de conservar la arquitectura de la mortaja. Una vez móvil el tobillo, se lleva a cabo la técnica quirúrgica como está descrita.

El paciente estuvo sin apoyo durante tres semanas y posteriormente marcha con muletas por una semana más. Hoy en día, a 20 meses de la cirugía, el paciente se encuentra con dorsiflexón de $20^{\circ}$ y flexión plantar de $25^{\circ}$; asimismo, puede caminar de forma recreativa, completamente satisfecho de haberse hecho la cirugía de prótesis total (Figura 5).

\section{DISCUSIÓN}

En la actualidad, aun en casos de OA de la subtalar, parece existir un consenso entre los expertos en tratar de preservar esta articulación, dado que al recuperar la función del tobillo, el dolor asociado a dicha articulación por lo regular disminuye considerablemente o, incluso, desaparece. Asimismo, al recuperar la dorsiflexión pasiva del tobillo en la paciente del primer caso, se pretende recuperar el recurvatum de rodilla, generado por el bloqueo en varo y equino del retropié de esta paciente. Markus Preis y colaboradores reportaron mejoría en los rangos de movimiento del retropié en su serie de desartrodesis con seguimiento a cinco años de $23^{\circ} \pm 7^{\circ}$ (dorsiflexión de $8.5^{\circ} \pm 3^{\circ}$ y flexión plantar de $15^{\circ} \pm$ $5^{\circ}$ ); además de que enfatizó que aquellos pacientes que estaban fijados en equino alcanzaron una dorsiflexión de al menos $5.8^{\circ}$.

Las opciones de tratamiento para la inestabilidad subastragalina están enfocadas en la reconstrucción del complejo ligamentario lateral de tobillo (especialmente el ligamento peroneo-calcáneo) o los ligamentos sub-talares. Muchas de estas técnicas implican el debilitamiento de los estabilizadores secundarios del tobillo (v.g. tendón peroneo corto) y el desgaste de estructuras óseas (v.g. astrágalo) por túneles que se practican durante dicha reconstrucción. ${ }^{8}$

Una alternativa para un paciente con pseudoartrosis de tobillo es la revisión de la misma a través de una artroplastia total. Las preocupaciones lógicas son la rehabilitación de la musculatura que hará funcionar este sistema (principalmente tibial anterior y tríceps); sin embargo, dado el tiempo entre la identificación de la pseudoartrosis y el reemplazo articular,
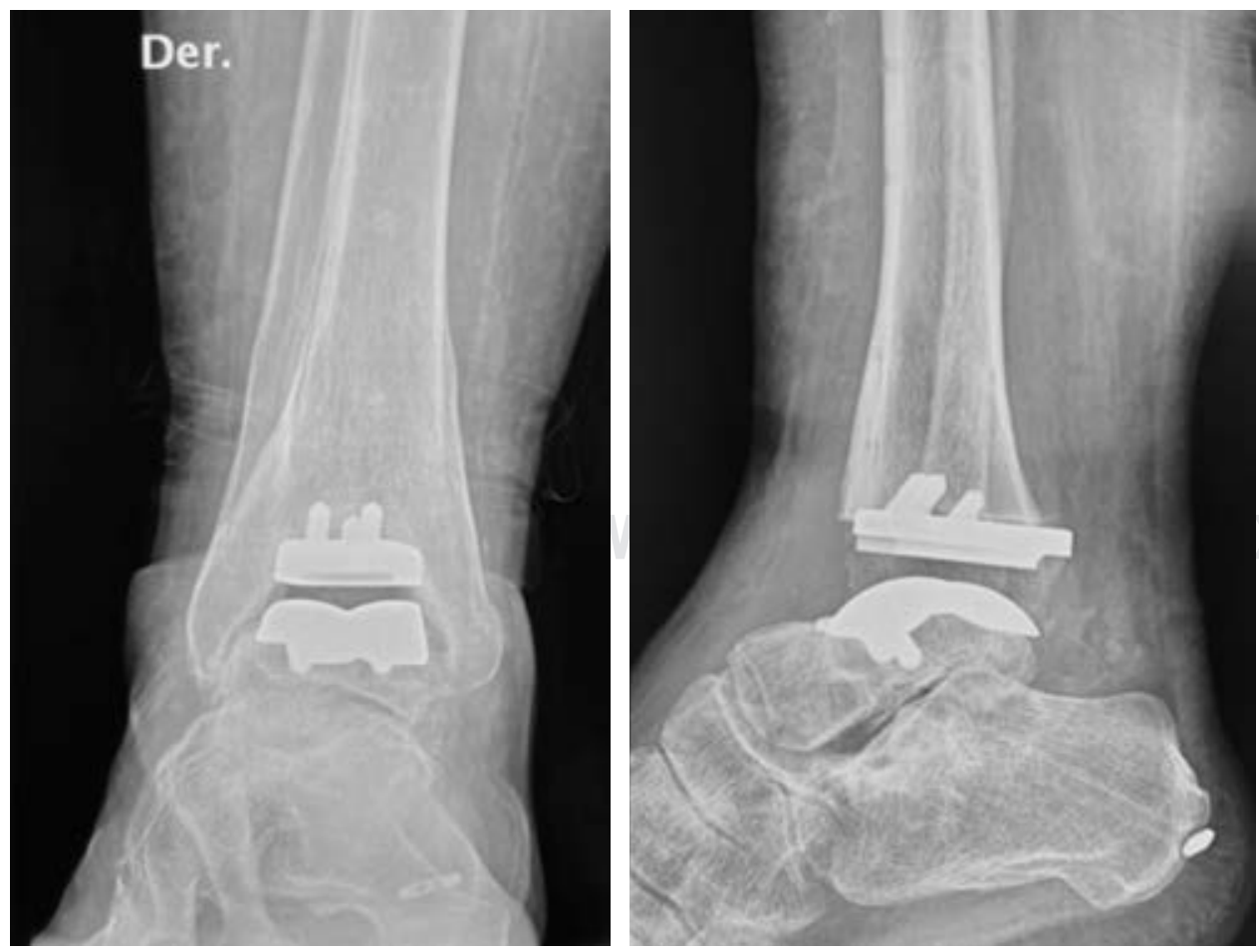

Figura 3:

Radiografía anteroposterior y lateral del tobillo posterior a la prótesis total y estabilización de la subastragalina. 
Figura 4:
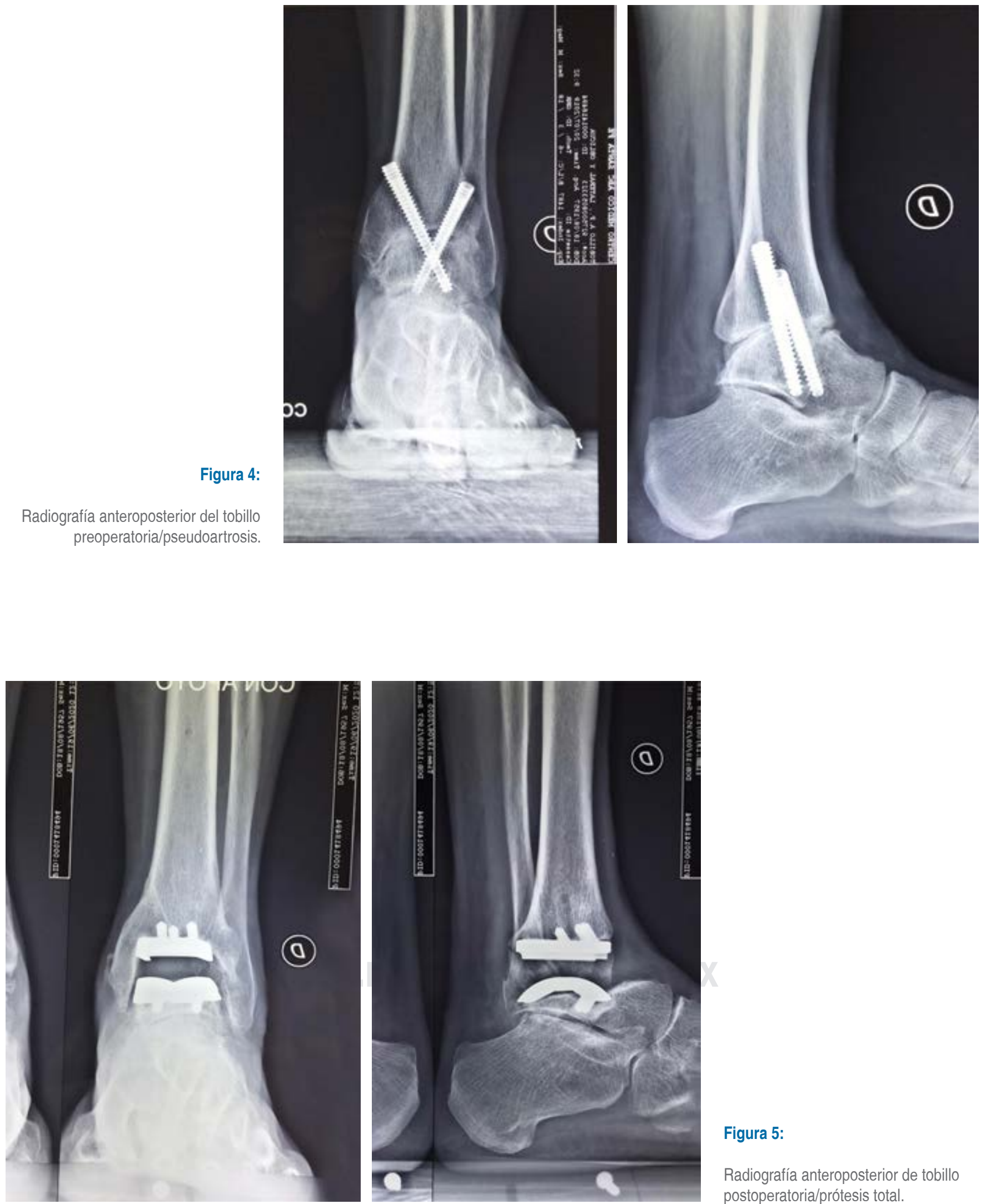

Figura 5:

Radiografía anteroposterior de tobillo postoperatoria/prótesis total. 
no hay evidencia de que esta «inutilidad» muscular dirija la evolución hacia un mal resultado funcional. ${ }^{15} \mathrm{El}$ paciente del caso 2 estuvo satisfecho funcionalmente por un año con la artrodesis y después de repetidas caminatas de larga distancia durante un viaje empezó a referir dolor constante. $\mathrm{Al}$ cabo de 20 meses, refiere poder caminar largas distancias con un mínimo dolor al final del día y estar satisfecho con el procedimiento.

En ambos casos se mostraron buenos resultados respecto a la ganancia en la dorsiflexión pasiva del tobillo, impactando en una mejor marcha e independencia y, por lo tanto, en una mejor calidad de vida de nuestros pacientes.

\section{CONCLUSIÓN}

De ninguna manera la artrodesis de tobillo se considera un procedimiento obsoleto, pues a lo largo del tiempo ha demostrado muy buenos resultados en el alivio del dolor del paciente y su regreso a la función. La diferencia es que la prótesis logra la preservación del movimiento con activación muscular intacta, lo que conlleva a una marcha muy cercana a lo fisiológico sin sobrecarga de las articulaciones vecinas. La decisión de hacer uno u otro procedimiento dependerá de la elección y pericia del cirujano, las condiciones del paciente (edad, alineación del tobillo/retropié, infección previa, necrosis avascular, etcétera).

La desartrodesis es un procedimiento muy demandante que requiere una óptima habilidad de parte del cirujano para ejecutarla. A pesar de esto, es un procedimiento que tiene el potencial de devolver al paciente la movilidad del tobillo y, como es de esperarse, una marcha con mejor cadencia, además de disminuir o eliminar el riesgo de la artrosis temprana de articulaciones vecinas por transferencia.

\section{REFERENCIAS}

1. Hayes BJ, Gonzalez T, Smith JT, Chiodo CP, Bluman EM. Ankle arthritis: you can't always replace it. J Am Acad Orthop Surg. 2016; 24 (2): e29-e38.

2. Barg A, Pagenstert GI, Hügle T, Gloyer M, Wiewiorski M, Henninger HB et al. Ankle osteoarthritis: etiology, diagnostics, and classification. Foot Ankle Clin. 2013; 18 (3): 411-426.

3. Payo J, Montiel V, Alfonso M, Villas C. Resultado funcional esperable tras una artrodesis bilateral de tobillo. Valoración de 3 casos y revisión de la literature. Rev Pie Tobillo. 2018; 32 (1): $35-42$.

4. Takakura Y, Tanaka Y, Kumai T, Tamai S. Low tibial osteotomy for osteoarthritis of the ankle. Results of a new operation in 18 patients. J Bone Joint Surg Br. 1995; 77 (1): 50-54.

5. Pagenstert G, Knupp M, Valderrabano V, Hintermann B. Realignment surgery for valgus ankle osteoarthritis. Op Orthop Traumatol. 2009; 21 (1): 77-87.

6. Valderrabano V, Hintermann B, Horisberger M, Fung TS. Ligamentous posttraumatic ankle osteoarthritis. Am J Sports Med. 2006; 34 (4): 612-620.

7. Jastifer JR, Gustafson PA. The subtalar joint: biomechanics and functional representations in the literature. Foot (Edinb). 2014; 24 (4): 203-209.

8. Keefe DT, Haddad SL. Subtalar instability. Etiology, diagnosis, and management. Foot Ankle Clin. 2002; 7 (3): 577-609.

9. Rao S, Ellis SJ, Deland JT, Hillstrom H. Nonmedicinal therapy in the management of ankle arthritis. Curr Opin Rheumatol. 2010; 22 (2): 223-228.

10. Morash J, Walton DM, Glazebrook M. Ankle arthrodesis versus total ankle arthroplasty. Foot Ankle Clin. 2017; 22 (2): 251-266.

11. Pellegrini MJ, Schiff AP, Adams Jr. SB, Queen RM, DeOrio JK, Nunley JA et al. Conversion of tibiotalar arthrodesis to total ankle arthroplasty. J Bone Joint Surg Am. 2015; 97 (24): 2004-2013.

12. Cody EA, Scott DJ, Easley ME. Total ankle arthroplasty: a critical analysis review. JBJS Rev. 2018; 6 (8): e8.

13. Hintermann B, Barg A, Knupp M, Valderrabano V. Conversion of painful ankle arthrodesis to total ankle arthroplasty. J Bone Joint Surg Am. 2009; 91 (4): 850-858.

14. Preis M, Bailey T, Marchand LS, Barg A. Can a threecomponent prosthesis be used for conversion of painful ankle arthrodesis to total ankle replacement? Clin Orthop Relat Res. 21017; 475 (9): 2283-2294.

15. Huntington WP, Davis WH, Anderson R. Total ankle arthroplasty for the treatment of symptomatic nonunion following tibiotalar fusion. Foot Ankle Spec. 2016; 9 (4): 330-335. 